\title{
Effect of polyoxyethylene sorbitan esters and sodium caseinate on physicochemical properties of palm-based functional lipid nanodispersions.
}

\begin{abstract}
The main objective of the present study was to investigate the effect of polyoxyethylene sorbitan esters and sodium caseinate on physicochemical properties of palm-based functional lipid nanodispersions prepared by the emulsification-evaporation technique. The results indicated that the average droplet size increased significantly $(\mathrm{P}<0.05)$ by increasing the chain length of fatty acids and also by increasing the hydrophile-lipophile balance value. Among the prepared nanodispersions, the nanoemulsion containing Polysorbate 20 showed the smallest average droplet size $(202 \mathrm{~nm})$ and narrowest size distribution for tocopheroltocotrienol nanodispersions, while sodium caseinate-stabilized nanodispersions containing carotenoids had the largest average droplet size $(386 \mathrm{~nm})$, thus indicating a greater emulsifying role for Polysorbate 20 compared with sodium caseinate.
\end{abstract}

Keyword: Polyoxyethylene sorbitan esters (Tween); Sodium caseinate; Emulsion properties; Functional lipid nanodispersions. 